\title{
Energy-Efficient Time-Stampless Adaptive Nonuniform Sampling
}

\author{
Soheil Feizi \\ RLE at MIT \\ Email: sfeizi@mit.edu \\ Georgios Angelopoulos \\ RLE at MIT \\ Email: georgios@mit.edu
}

\author{
Vivek K Goyal \\ RLE at MIT
}

Email:vgoyal@mit.edu

\author{
Muriel Médard \\ RLE at MIT
}

Email: medard@mit.edu

\begin{abstract}
Proposal Summary-Nowadays, since more and more batteryoperated devices are involved in applications with continuous sensing, development of an efficient sampling mechanisms is an important issue for these applications. In this paper, we investigate power efficiency aspects of a recently proposed adaptive nonuniform sampling. This sampling scheme minimizes the energy consumption of the sampling process, which is approximately proportional to sampling rate. The main characteristics of our method are that, first, sampling times do not need to be transmitted, since the receiver can compute them by using a function of previously taken samples, and second, only innovative samples are taken from the signal of interest, reducing the sampling rate and therefore the energy consumption. We call this scheme Time-Stampless Adaptive Nonuniform Sampling (TANS). TANS can be used in several scenarios, showing promising results in terms of energy savings, and can potentially enable the development of new applications that require continuous signals sensing, such as applications related to health monitoring, location tracking and entertainment.
\end{abstract}

\section{INTRODUCTION}

In the next few years, the development of a new era is expected to be launched where mobile phones, sensors and other lightweight and battery-operated devices will be involved in human's everyday life, affecting their choices as well as their emotions. These devices have already started to become more and more intelligent, equipped with extraordinary features. They are actually considered to be able to continuously interact with the human's surrounding environment, enabling the development of new applications in health monitoring, social networking, entertainment and several other domains, enhancing human's quality of life.

However, since the majority of these devices are resourceconstrained with strict power budgets, powered by small batteries or even by energy scavenging, reducing their power consumption would be extremely beneficial for their battery lifetime. Proposed applications use these devices to continuously take measurements of different quantities such as voice, acceleration, light or voltage levels. Then, after processing these quantities and extracting required features, they communicate with humans or other devices. A main concern in all of these applications is the high power consumption of continuously sampling, processing and transmitting information. In this work, we propose a general scheme for efficiently sampling signals in an adaptive way, based on a recently proposed sampling framework [1]. This sampling scheme minimizes

This material is partially supported by IFC research program for connectivity, the Georgia Institute of Technology Under Award Number: 017894-010. the required sampling rate and therefore the energy consumed during the sampling process, which can be a significant part of the system's energy consumption in many applications [2].

For band-limited deterministic signals, this problem is wellstudied. For example, the Nyquist sampling theorem proposes an appropriate uniform sampling setup which leads to a reconstruction with zero error. There are some level-crossing based nonuniform sampling scheme with iterative decoders (as examples, see [3] and [4]). Reference [5] proposes two adaptive sampling schemes for band-limited deterministic signals. These schemes are based on some definitions of local bandwidth by using linear time-varying low pass filters [6], and time-warping of bandlimited signals [7].

Although the Nyquist sampling theorem proposes an appropriate uniform sampling setup for band-limited deterministic signals, some taken samples may be redundant because the maximum bandwidth of the signal may not be a good measure of signal variations at different times. An ideal approach would be to take samples only when they are innovative for the considered application. This leads to an adaptive nonuniform sampling scheme.

There are two difficulties in a traditional nonuniform sampling scheme: first, determining the next sampling time step at each time (i.e., rate control), and second, storing or transmitting these sampling times, since they are required in the reconstruction process. Here, we use a framework proposed in [1] to deal with these problems. This new adaptive nonuniform sampling framework has two key characteristics: first, it takes samples only when they are innovative for the considered application and consequently reduces the number of required measurements. Second, unlike traditional nonuniform sampling procedures, sampling times do not need to be transmitted since the receiver can recover them in our framework, which in turn saves transmission rate and power. We call our scheme Time-Stampless Adaptive Nonuniform Sampling (TANS). TANS can be used in several applications.

Th rest of the paper is organized as follows. In section II, we revisit TANS framework of reference [1] and compare its energy efficiency aspects with other related schemes. In section III, we show that, TANS can be used widely in modern systems providing significant rate and power benefits. Specially, we consider applications of TANS on health monitoring and perform a case-study analysis on its energy consumption for sampling ECG signals. We conclude the paper in Section IV 


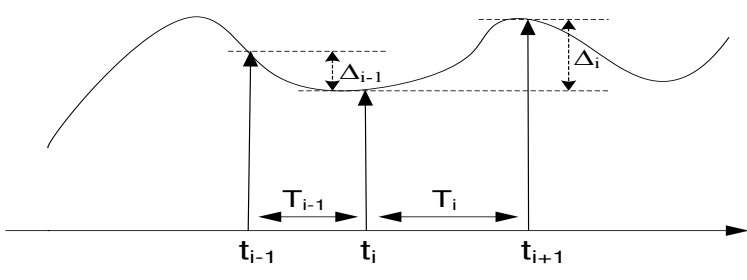

Fig. 1. TANS setup

\section{Proposed FrameWORK AND RELATED Work}

Taking samples from a signal satisfying some requirements on the sampling rate and the reconstruction error is one of the main problems in various applications. Having redundant samples costs extra power consumption in the sampling procedure and also in afterward processes. For example, if these samples are needed to be transmitted somewhere else, having lots of samples results in higher transmission energy or in extra energy spent for compressing these samples. Hence, a smart sampling scheme should take samples as needed for the considered application (i.e., innovative samples). This leads to an adaptive sampling scheme which inherently is nonuniform.

Some nonuniform adaptive sampling schemes have been proposed in the literature. For instance, a nonuniform sampling scheme based on level-crossings with iterative decoding is considered in [3], while reference [4] presents an approach based on level crossings with a filtering technique, which adapts the sampling rate and filter order by analyzing the input signal variations online. Also, two adaptive sampling schemes for band-limited deterministic signals are proposed in [5]. These schemes are using some definitions of local bandwidth, based on linear time-varying low pass filters [6], and time-warping of band-limited signals [7].

There are two issues about these nonuniform sampling schemes which make them difficult to be applied in practical applications: first, they are designed for specific signal models (i.e., they are not generic), and second, sampling times are needed to be kept or transmitted in order to be used in the reconstruction process. In this work, we develop a new framework for adaptive nonuniform sampling which solves these two issues. The key idea of this framework is that, time intervals between samples can be computed by using a function of previously taken samples. Therefore, keeping sampling times (time-stamps), except initialization times, is not necessary. The aim of this sampling framework is to have a balance between the reconstruction distortion and the average sampling rate. We call the function by which sampling time intervals can be computed sampling function and we refer to this sampling framework as Time-Stampless Adaptive Nonuniform Sampling (TANS).

This sampling structure can be applied appropriately on different signal models such as deterministic or stochastic, and continuous or discrete signals, and for each, a different sampling function can be derived. However, in this proposal, we explain the framework for continuous signals. To explain

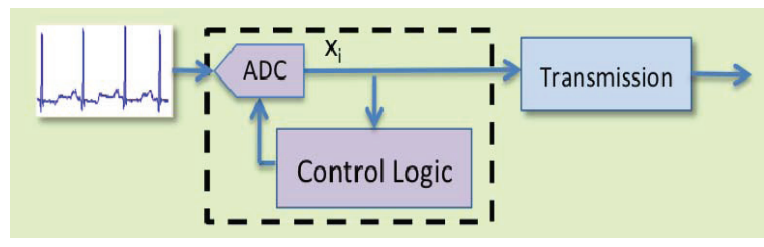

(a)

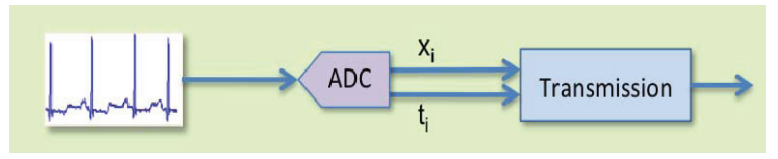

(b)

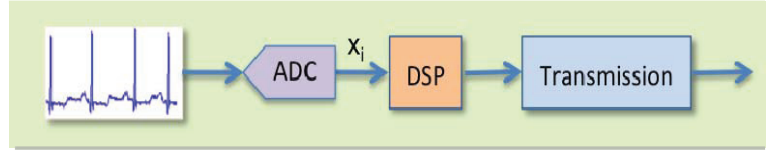

(c)

Fig. 2. (a) Block diagram of the proposed nonuniform sampling framework. The control logic implements the sampling function and determines sampling times. (b) A traditional Nonuniform sampling scheme. (c) A traditional uniform sampling scheme.

this sampling framework, we borrow some definitions and notations from reference [1].

Consider a continuous signal $X(t)$. Suppose the $i^{t h}$ sample is taken at time $t_{i}$. Define $T_{i} \triangleq t_{i+1}-t_{i}$ and $\Delta_{i} \triangleq X\left(t_{i+1}\right)-$ $X\left(t_{i}\right)$. Then, we take the $(i+1)^{t h}$ sample after a time interval of length $T_{i}=f\left(\bigcup_{j=i-m+1}^{i-1}\left\{T_{j}, \Delta_{j}\right\}\right) . f($.$) is the sampling$ function. Since the next sampling time step is a function of $m$ most recently taken samples, we say the order of the sampling function $f($.$) is m$ (Figure 1 and 2). The sampling function has to be known on both sampling and reconstruction sides.

This sampling structure is nonuniform except in trivial cases when the sampling function is a constant-valued function. However, the key characteristic of our approach is that, unlike traditional nonuniform sampling procedures, keeping sampling times (time-stamps) is not necessary in our framework, because, these times can be recovered by using the sampling function and previously taken samples. In the above example, we have $t_{i+1}=t_{i}+f\left(\bigcup_{j=i-m+1}^{i-1}\left\{T_{j}, \Delta_{j}\right\}\right)$. Note that, first $m$ sampling times should be kept to initialize the process. However, its rate effect is negligible when the number of samples increases.

In the above case, TANS is causal because the next sampling time depends on samples taken before that time. In general, it can be designed to be non-causal. TANS is an adaptive process which learns from the signal through the taken samples, because the sampling function depends on local characteristics of the signal. Finding an appropriate sampling function of TANS is at the heart of our schemes and depends on different considered applications with various sampling requirements such as the sampling rate, the distortion requirement, the computational complexity etc. 
One may note that, for a discrete stochastic signal, one naive scheme is to take samples uniformly at a fixed high rate using an $\mathrm{ADC}$, and then, use source coding to compress these samples approximately to their entropy rate before transmission, as shown in Fig. 2-c. While this technique is theoretically optimal, it has some inefficiencies in practice in terms of the extra sampling and processing power, compared to a scheme which takes samples when they are innovative, since the power consumed during the sampling process depends linearly on the sampling frequency. Moreover, to have an appropriate performance, long blocks of samples are needed to be able to use source coding efficiently, especially if statistical properties of the signal vary slowly in time. This block-based approach may lead to a large delay on the reconstruction side. Instead, our proposed scheme can be viewed as a real-time delay-free compression scheme. It adaptively compresses the signal by using its local properties causally and hence, reduces the power consumption.

\section{Applications OF TANS}

Sensing is a process performed in any system interacting with the real world and one of the main power consumption contributors of low power devices. For this reason, we believe that our framework can be applied to a wide variety of different systems, enabling the development of new applications in the future. In the next paragraphs, we illustrate our proposed framework in only two cases, however, TANS can also be used in several other scenarios.

\section{A. Sensing in Health Monitoring}

Several research projects refer to continuous health monitoring by using either custom sensors or platforms based on commercial mobile phones, recording ECG signals and other human's vital information. The current approach for the design of these systems is to continuously sample the considered signals, at a rate greater than or equal to the maximum rate of happening an event of interest. However, in our proposed approach, the sampling rate is adapted by using previously taken samples as described in Section II. We also do not need to keep sampling times. Hence, our scheme can achieve a lower power consumption in the sensing process.

For example, a generic sampling function can be designed by bounding the derivative of the signal by the integral of its Fourier transform and then using the Cauchy-Schwarz inequality as follows: $T_{i}=\left(\frac{p}{c} \frac{1}{w\left(t_{i}\right)^{2}}\right)^{1 / 3}$, where $p$ is the signal power, $c$ is a sampling parameter and $w\left(t_{i}\right)$ is an approximation of the derivative of the signal at time $t_{i}$ (i.e., $\left.w\left(t_{i}\right)=\left|\frac{\Delta_{i-1}}{T_{i-1}}\right|\right)$. Note that, the higher the derivative at time $t_{i}$, the higher the signal variations at this time, and the smaller the sampling step size. Note that, this sampling function is not designed specifically for ECG signals. In our early simulations on ECG signals, we use the following sampling function:

$$
T_{i}=\left\{\begin{array}{cl}
T_{i-1}+c_{1} & w\left(t_{i}\right)<t h_{1} \text { and } T_{i}>T^{\text {min }} \\
T_{i-1} & t h_{1} \leq w\left(t_{i}\right)<t h_{2} \\
T_{i-1}-c_{2} & w\left(t_{i}\right) \geq t h_{2} \text { and } T_{i}<T^{\text {max }}
\end{array}\right.
$$

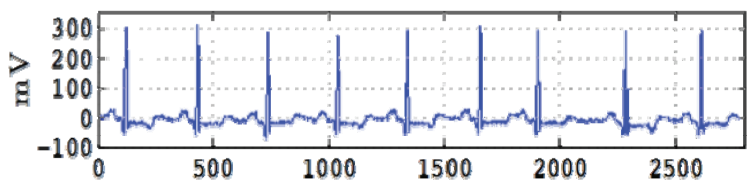

(a)

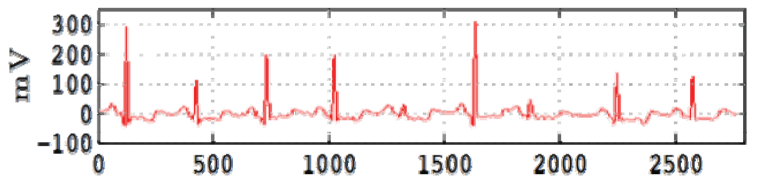

(b)

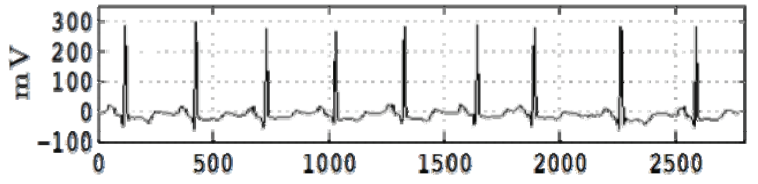

(c)

Fig. 3. Reconstructed signals using: (a) Uniform sampling with a sampling rate of $1000 \mathrm{samples} / \mathrm{sec}$, (b) Uniform sampling with a sampling rate of 100 samples/sec, and (c) ETANS sampling with an average sampling rate of 100 samples/sec.

Here, $w\left(t_{i}\right)$ is a measure of local bandwidth which is determined by using characteristics of ECG signals. $c_{1}, c_{2}$, $T^{\min }$ and $T^{\max }$ are sampling parameters where by using them the reconstruction error and the average sampling rate can be adjusted. Our simulation results with real ECG signals [8] indicate advantages of our proposed approach, reducing by approximately an order of magnitude the sampling rate compared to uniform sampling.

Figure 3-a shows the reconstructed signal by using uniform sampling with a sampling rate of 1000 samples/sec. Figure 3-b shows a similar figure for a uniform sampling scheme with a sampling rate of 100 samples/sec. Finally, Figure 3-c illustrates the reconstructed signal by using TANS with an average sampling rate of $100 \mathrm{samples} / \mathrm{sec}$. Comparing reconstruction errors show that, our approach provides a better reconstructed signal given the same sampling rate (Figures 3-(b) and (c)).

Table III-B shows a case-study analysis of energy consumption of three schemes (uniform sampling, compressive sensing [9] and TANS) for ECG signal sampling. As demonstrated in this table, TANS is more power efficient than other schemes.

\section{B. Sensing in Smartphones}

Our framework can also be applied to the majority of applications that involve the interaction of smartphones with the human surrounding environment to facilitate his life. For instance, the use of smartphones, equipped with several sensors has been proposed for traffic monitoring [10], sound sensing [11], location tracking [12] and many other scenarios. Although most of these proposed ideas seem promising applications in the future, the strict power constraints of portable devices are a main obstacle in their realizations. 
TABLE I

A CASE-STUDY ENERGY ANALYSIS FOR PROCESSING A 10-SEC EKG SIGNAL

\begin{tabular}{|c||c|c|c|}
\hline & Uniform Sampling & Compressive Sensing & TANS \\
\hline \hline ADC [13] & $16 \mathrm{uJ}$ & $16 \mathrm{uJ}$ & $1.6 \mathrm{uJ}$ \\
\hline Compression & $1.7 \mathrm{~mJ}[14]$ & $19 \mathrm{uJ}[9]$ & 0 \\
\hline Extra Logic & 0 & 0 & $\approx 5 \mathrm{uJ}$ \\
\hline Total & $1.71 \mathrm{~mJ}$ & $35 \mathrm{uJ}$ & $6.6 \mathrm{uJ}$ \\
\hline
\end{tabular}

We believe that, our framework can be an efficient solution in applications requiring continuous sampling and interaction with the real world, since, as reported in [2], sensors in a modern smartphone can consume approximately up to $60 \%$ of the overall power consumption when the phone is in sleep mode.

\section{CONCLUSIONS}

In this paper, we investigated power efficiency aspects of a recently introduced sampling framework called TANS (TimeStampless Adaptive Nonuniform Sampling). This framework minimizes the required sampling rate and therefore energy consumption of the sampling process. In this framework, time intervals between samples can be computed by using a function of previously taken samples, called a sampling function. Hence, though it is a nonuniform sampling scheme, we do not need to keep sampling times. TANS has two key characteristics: first, it takes samples only when they are innovative for the considered application and consequently reduces the number of required measurements. Second, unlike traditional nonuniform sampling procedures, sampling times do not need to be kept or transmitted since the receiver can recover them by using the sampling function and previously taken samples, which in turn saves some transmission rate and power. TANS can be used in several applications. Here, we showed that, this framework can be used widely in modern systems such as health monitoring providing significant energy benefits.

\section{REFERENCES}

[1] S. Feizi, V. K. Goyal, and M. Médard, "Locally adaptive sampling," in Communication, Control, and Computing (Allerton), 2010 48th Annual Allerton Conference on. IEEE, 2010, pp. 152-159.

[2] B. Priyantha, D. Lymberopoulos, and J. Liu, "Enabling energy efficient continuous sensing on mobile phones with littlerock," in Proceedings of the 9th ACM/IEEE International Conference on Information Processing in Sensor Networks, ser. IPSN '10, 2010, pp. 420-421.

[3] F. Marvasti, Nonuniform sampling: Theory and Practice. Plenum Publishers Co., 2001.

[4] L. F. S. M. Qaisar and M. Renaudin, "Computationally efficient adaptive rate sampling and filtering," in 15th European Signal Processing Conference (EUSIPCO 07), Poznan, Poland, Sep. 2007, pp. 2139-2143.

[5] D. Wei, "Sampling based on local bandwidth," in Master Thesis, MIT, 2007.

[6] T. A. C. M. Claasen and W. F. G. Mecklenbrauker, "On stationary linear time-varying systems," IEEE Transactions on Circuits and Systems, vol. 29, no. 3, pp. 169-184, 1982.

[7] K. Horiuchi, "Sampling principle for continuous signals with timevarying bands," Information and Control, vol. 13, no. 1, pp. 53-61, 1968.

[8] A. L. Goldberger and et al., "Physiobank, physiotoolkit, and physionet: Components of a new research resource for complex physiologic signals," Circulation, vol. 101, no. 23, pp. e215-e220, 2000 (June 13).
[9] F. Chen, A. Chandrakasan, and V. Stojanovic, "A signal-agnostic compressed sensing acquisition system for wireless and implantable sensors," in Custom Integrated Circuits Conference (CICC), 2010 IEEE. IEEE, pp. 1-4.

[10] B. Hoh, M. Gruteser, R. Herring, J. Ban, D. Work, J.-C. Herrera, A. M. Bayen, M. Annavaram, and Q. Jacobson, "Virtual trip lines for distributed privacy-preserving traffic monitoring," in Proceeding of the 6th international conference on Mobile systems, applications, and services, ser. MobiSys '08, 2008, pp. 15-28.

[11] H. Lu, W. Pan, N. D. Lane, T. Choudhury, and A. T. Campbell, "Soundsense: scalable sound sensing for people-centric applications on mobile phones," in Proceedings of the 7th international conference on Mobile systems, applications, and services, ser. MobiSys '09, 2009, pp. 165-178.

[12] F. Bagci, F. Kluge, T. Ungerer, and N. Bagherzadeh, "Optimisations for locsens an indoor location tracking system using wireless sensors," Int. J. Sen. Netw., vol. 6, pp. 157-166, November 2009.

[13] S. Jocke, J. Bolus, S. Wooters, A. Jurik, A. Weaver, T. Blalock, and B. Calhoun, "A 2.6- $\mu \mathrm{w}$ sub-threshold mixed-signal ecg soc," in $V L S I$ Circuits, 2009 Symposium on. IEEE, 2009, pp. 60-61.

[14] E. Chua, C. Fu, and W. Fang, "VLSI implementation of a mixed bio-signal lossless data compressor for portable brain-heart monitoring systems," in Consumer Electronics (ICCE), 2011 IEEE International Conference on. IEEE, pp. 557-558. 